\title{
镍催化不对称扩环反应制备含硅手性中心苯并噻咯
}

\author{
尹官武徐利文*
}

(杭州师范大学材料与化学化工学院 有机硅化学及材料技术教育部重点实验室 杭州 311121)

\section{Access to Enantioenriched Silicon-Stereogenic Benzosiloles via Nickel(0)-Catalyzed Asymmetric Ring Expansion}

\author{
Yin, Guanwu $\quad \mathrm{Xu}$, Liwen*
}

(Key Laboratory of Organosilicon Chemistry and Material Technology, Ministry of Education, College of Material, Chemistry and Chemical Engineering, Hangzhou Normal University, Hangzhou 311121)

含硅的 $\pi$ 电子共轭体系分子凭借其在荧光材料和电 子传输领域所展示的优良性能, 已在材料科学领域引起 了广泛的关注 ${ }^{[1]}$. 与传统的碳骨架 $\pi$ 电子共轭体系相比, 硅原子的替代会导致 $\pi$ 电子共轭体系能级发生变化, 进 而提高体系的堆积能力、结晶性以及电荷流动性 ${ }^{[2]}$. 苯 并噻咯作为常见的一种含硅的 $\pi$ 电子共轭体系骨架分 子, 由于其独特的电子及光学性质, 已被逐渐应用在有 机半导体领域中 ${ }^{[3]}$. 近年来, 对于构建不同结构的苯并 噻咯类化合物的方法已逐渐成熟, 然而对于合成含硅手 性中心的苯并噻咯化合物却仍然具有较大的挑战.

2012 年, Chatani 课题组 ${ }^{[4]}$ 通过铑催化碳-硅键活化 的策略实现合成了硅手性中心的苯并噻咯(Scheme 1a). 随后, 徐利文 ${ }^{[5]}$ 和何川课题组 ${ }^{[6]}$ 分别利用去对称的方法 以及不对称脱氢的碳氢硅烷化反应, 通过铑催化不对称 合成一系列具有硅手性中心的苯并噻咯分子(Schemes $1 \mathrm{~b}, 1 \mathrm{c})$. 然而, 以上方法均采用了价格昂贵的铑金属催 化剂, 并且在底物的普适性和产物结果的多样性上都存 在一定的局限性. 因此开发一种丰产金属催化策略, 从 而高效、高对映选择性地合成硅手性中心的苯并噻咯化 合物, 对于满足日益增长的手性 $\pi$ 电子共轭材料的合成 需求具有重要的意义.

硅杂环丁烷在过渡金属催化作用下通过扩环反应 得到一系列含有硅手性中心的硅杂环化合物的方法 ${ }^{[7]}$ 已 有报道. 受此启发, 近日, 南开大学化学学院赵东兵课 题组 ${ }^{[8]}$ 在苯乙烯的邻位创新性地引入含有不同取代基的 硅杂环丁烷, 并基于过渡金属镍催化的扩环反应, 高效 地合成了一系列具有硅手性中心的苯并噻咯分子. 该方
法采用镍催化体系高对映选择性地构建了含有硅手性 中心的苯并噻咯分子, 并且具有良好的底物普适性, 同 时反应转化率为 $100 \%$, 具有很好的原子经济性.

(1) Pervious works

(a)

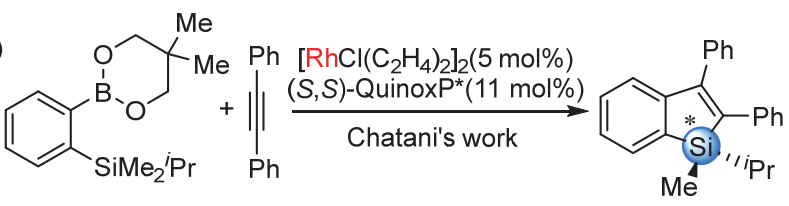

(b)

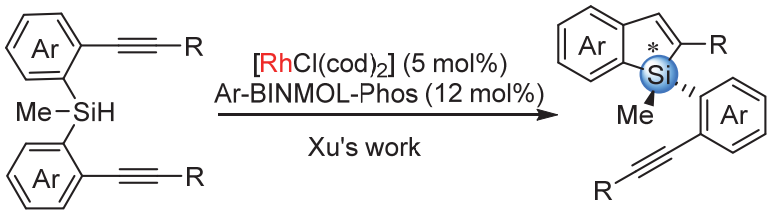

(c)
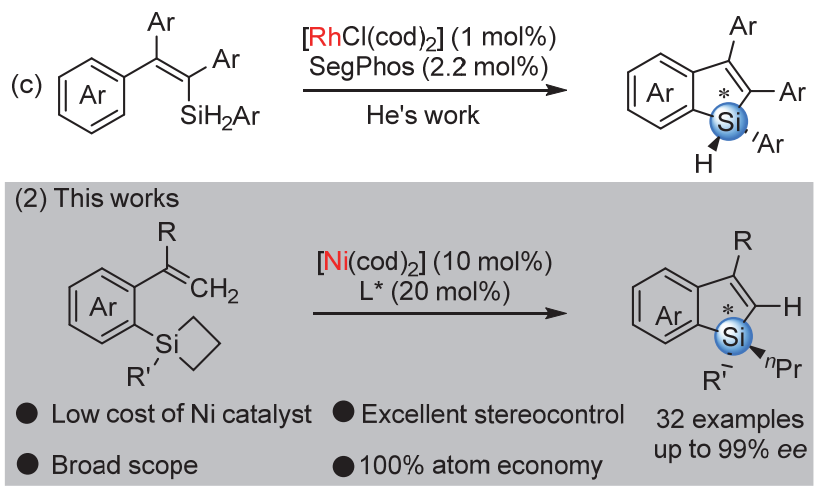

图式 1 合成硅手性中心苯并噻咯的不对称催化方法 Scheme 1 Access to silicon-stereogenic benzosiloles with catalytic enantioselective approach

为建立高效催化体系, 赵东兵等 ${ }^{[8]}$ 利用过渡金属镍 作为催化剂对反应条件进行篮选, 确立了 $\mathrm{Ni}(\mathrm{cod})_{2}$ 和

* Corresponding author. E-mail: liwenxu@hznu.edu.cn. Published online November 30, 2021. 
$\mathrm{PCy}_{3}$ 所组成的催化剂在甲苯中反应 $12 \mathrm{~h}$ 为最佳反应条 件, 并在消旋的条件下进行了底物拓展(Scheme 2). 反 应结果表明，当合成 3-位含有取代的苯并噻咯时，可用 $\mathrm{PCy}_{3}$ 作为配体, 而合成 2,3-位无取代基的苯并噻咯时, 则需要采用 $\mathrm{P}^{n} \mathrm{Pr}_{3}$ 作为配体. 为了合成含硅手性中心的 苯并噻咯化合物, 作者又考察了一系列手性膦配体, 并 发现当向反应体系中加入手性磷配体后, 反应速率普遍 降低，只有汤文军课题组 ${ }^{[9]}$ 开发的单膦配体可以高效地 不对称催化该反应. 针对此弊端作者在反应体系中加入 了催化量的叔丁醇钠, 并以高产率和对应选择性得到了 硅手性苯并噻咯, 随后, 作者对底物进行了拓展, 并发 现苯乙烯 $\alpha$ 位的取代基位阻大小对产物的 $e e$ 值有一定 影响.<smiles>[R]C(=C)c1ccccc1[Si]1([R])CCC1</smiles>
$\mathrm{R}^{\prime}$

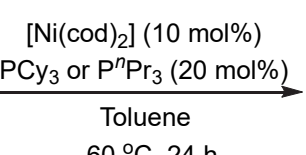

$60^{\circ} \mathrm{C}, 24 \mathrm{~h}$

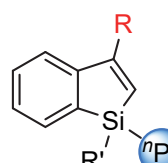
$\mathrm{R}^{\prime} \mathrm{Y} \operatorname{Pr}$<smiles>CCC[SiH]1C=C(C)c2ccccc21</smiles><smiles></smiles><smiles>CCCCCCCCC</smiles>
$\mathrm{H}_{3} \mathrm{C}^{\prime} \mathrm{n} \mathrm{Pr}$

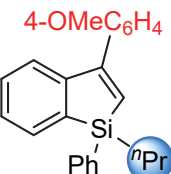<smiles>C=C[Si]1(C)C=Cc2ccccc21</smiles>

图式 2 通过扩环反应制备硅中心苯并噻咯

Scheme 2 Synthesis of silicon-stereogenic benzosiloles with ring expansion

为了进一步体现该方法的价值, 作者合成了具有双 硅桥联 $\pi$ 电子共轭体系的化合物 32 和 33 (Scheme 3), 并 对其光物理性能进行了表征. 由于具有大的 $\pi$ 电子共轭 体系, 化合物 32 和 33 在固体和溶液状态均在紫外光照 下表现出较强的蓝色荧光, 展示出了良好的荧光发射性 能. 在圆二色光谱中可以发现, 两组互为镜像的光谱, 由于化合物 33 比化合物 32 具有更大的 $\pi$ 电子共轭体系, 因此化合物 33 展示了更强的科顿效应. 在圆偏振光谱 测试中, 化合物 33 显示出一定强度的圆偏振光(CPL)信 号, 而化合物 32 没有明显的 CPL 信号. 为了进一步探 索反应机理，该团队针对此反应进行了氛代实验. 经核 磁检测发现, 与硅相连的丙基端位出现氝信号, 证明反 应经历了一个 $\beta-\mathrm{H}$ 消除的过程, 并提出了两种可能的催
化循环过程.

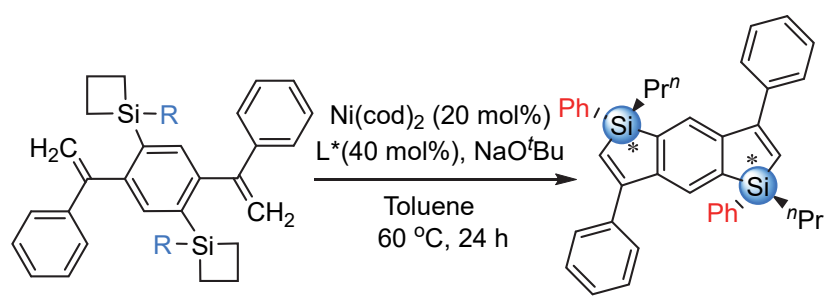

$\mathrm{R}=\mathrm{Me}$ or $\mathrm{Ph}$

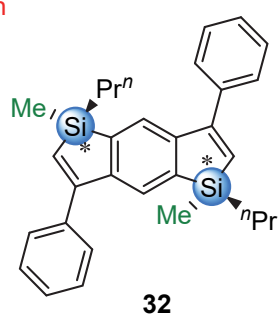

$94 \%, 99 \%$ ee
图式 3 高效构建具有硅含手性中心且具有双硅桥联 $\pi$-电子 体系的苯并噻咯

Scheme 3 Construction of enantioenriched silicon-stereogenic benzosiloles with the bis silicon-bridged $\pi$-extended systems

总之, 赵东兵课题组报道了一例镍催化合成硅手性 中心苯并噻咯分子的新反应过程, 该工作基于硅杂环丁 烷的扩环反应，实现了高效、高选择性地构建含有硅手 性中心的苯并噻咯分子, 成功构筑了具有苂光性能的双 硅桥联 $\pi$ 电子共轭体系化合物的合成, 并且有望在苂光 材料和电子传输领域发挥重要作用.

\section{References}

[1] Yang, X.; Zhou, G.; Wong, W.-Y. Chem. Soc. Rev. 2015, 44, 8484;

[2] Qin, Y.; Chen, H.; Yao, J.; Zhou, Y.; Cho, Y.; Zhu, Y.; Qiu, B.; Ju, C.-W.; Zhang, Z.-G.; He, F.; Yang, C.; Li, Y.; Zhao, D. Nat. Commun. 2020, 11, 5814 .

[3] Zhao, Z.; He, B.; Tang, B. Z. Chem. Sci. 2015, 6, 5347.

[4] Onoe, M.; Baba, K.; Kim, Y.; Kita, Y.; Tobisu, M.; Chatani, N. J. Am. Chem. Soc. 2012, 134, 19477.

[5] Tang, R.-H.; Xu, Z.; Nie, Y.-X.; Xiao, X.-Q.; Yang, K.-F.; Xie, J.-L.; Guo, B.; Yin, G.-W.; Yang, X.-M.; Xu, L.-W. iScience 2020, 23, 101268.

[6] Yuan, W.; You, L.; Lin, W.; Ke, J.; Li, Y.; He, C. Org. Lett. 2021, 23, 1367.

[7] Wang, X.-B.; Zheng, Z.-J.; Xie, J.-L.; Gu, X.-W.; Mu, Q.-C.; Yin, G.-W.; Ye, F.; Xu, Z.; Xu, L.-W. Angew. Chem., Int. Ed. 2020, 59, 790.

[8] Zhang, J.; Yan, N.; Ju, C.-W.; Zhao, D. Angew. Chem., Int. Ed. 2021, 60, 25723.

[9] Fu, W.; Tang, W. ACS Catal. 2016, 6, 4814. 